\title{
High Charge Storage Capacity Electrodeposited Iridium Oxide Film on Liquid Crystal Polymer-Based Neural Electrodes
}

\author{
Soowon Shin, ${ }^{1,2}$ Junghoon Kim,,$^{1,2}$ Joonsoo Jeong,,${ }^{1,2}$ Tae Mok Gwon, ${ }^{1,2}$ \\ Gwang Jin Choi, ${ }^{1,2}$ Sung Eun Lee, ${ }^{1,2}$ Jinhyung Kim, ${ }^{3,4}$ \\ Sang Beom Jun, ${ }^{5,6}$ Jin Woo Chang, ${ }^{3}$ and Sung June Kim ${ }^{1,2^{*}}$ \\ ${ }^{1}$ Department of Electrical and Computer Engineering, Seoul National University, \\ 1, Gwanak-ro, Gwanak-gu, Seoul, Korea \\ 2Inter-University Semiconductor Research Center, Seoul National University, \\ 1, Gwanak-ro, Gwanak-gu, Seoul, Korea \\ ${ }^{3}$ Department of Neurosurgery, Severance Hospital, Yonsei University, \\ 50, Yonsei-ro, Seodaemun-gu, Seoul, Korea \\ ${ }^{4}$ Department of Biological Science, Korea Advanced Institute of Science and Technology (KAIST), \\ 291, Daehak-ro, Yuseong-gu, Daejeon, Korea \\ ${ }^{5}$ Department of Electronics Engineering, Ewha Womans University, \\ 52, Ewhayeodae-gil, Seodaemun-gu, Seoul, Korea \\ ${ }^{6}$ Department of Brain and Cognitive Sciences, Ewha Womans University, \\ 52, Ewhayeodae-gil, Seodaemun-gu, Seoul, Korea
}

(Received November 7, 2015; accepted October 27, 2015)

Keywords: electrodeposited iridium oxide film, liquid crystal polymer, polymer-based neural prosthesis, flexible neural interface

This research presents the development of neural electrodes with a high charge storage capacity using electrodeposited iridium oxide film (EIROF). The neural electrode was fabricated on a flexible liquid crystal polymer (LCP) substrate, which has a low moisture absorption rate and a low moisture permeability. On the electrode sites, iridium oxide was electrodeposited to increase the charge storage capacity for facilitating both neural stimulation and neural recording. Although EIROFs have been previously proposed as an electrode material, the fabrication process was not thoroughly investigated and optimized. ${ }^{(20)}$ After electrodeposition using different numbers of rectangular voltage pulses and triangular waveforms, the iridium oxide electrodes were characterized in terms of charge storage capacity and electrochemical impedance. The surfaces of EIROFs were examined using atomic force microscopy (AFM) and scanning electron microscopy (SEM). In addition, the elementary composition of the EIROF surfaces was quantitatively determined using X-ray photoelectron spectroscopy (XPS). The in vivo neural recording from the rat ventral poster lateral (VPL) nucleus successfully showed that the proposed electrode is feasible for recording the neural activity.

${ }^{\bar{*}}$ Corresponding author: e-mail: kimsj@snu.ac.kr 


\section{Introduction}

Neural prostheses use electrical stimulation for restoration and control of the nervous system. For instance, cochlear implants and retinal implants deliver electrical stimulations to the neural pathways of people with auditory and visual impairments, respectively. ${ }^{(1-6)}$ Deep brain stimulation (DBS) also uses electrical stimulation to treat neuropathic disorders such as Parkinson's disease, epileptic seizures, and neuropathic pain. ${ }^{(7-9,34-38)}$

Although silicon has been the most widely used material for microelectrode arrays, polymerbased microelectrode arrays have been recently highlighted since a flexible substrate was preferred for long-term implantation. . $^{(2,9,11-15,18-20,24-26)}$ Various biocompatible polymer substrates have been employed, such as polyimide, parylene-C, and liquid crystal polymers (LCP). Among them, LCP is known to absorb much less moisture compared to other polymers, which is advantageous for long term durability in vivo. ${ }^{25-27)}$ Furthermore, it was reported that LCP-based electrodes can be fabricated via thin-film processes and thermal lamination process, allowing flexibility for various electrode designs. ${ }^{(2,9,12)}$ For these reasons, LCP is becoming an attractive substrate material for neural prostheses. ${ }^{(2,9,12,25-27,43)}$

Various materials have been tried as electrode materials for neural prostheses, such as platinum, gold, iridium oxide, poly(3,4-ethylenedioxythiophene):PEDOT, and titanium nitride. ${ }^{(10)}$ Noble metals such as platinum and gold have been widely used for electrode sites due to their high durability. However, microelectrodes with noble metals have insufficient charge-injection capacities for stimulation. Therefore, in order to increase the charge-injection capacity, materials with specific surface structures or with electrochemically non-polarizable characteristics have been developed, such as titanium nitride, PEDOT, and iridium oxide. The high charge-injection capacities of these materials are obtained due to their high surface roughness characteristics, making their electrochemical surface area (ESA) much higher than the geometric surface area (GSA). Titanium nitride is a capacitive charge-injection material, which is more desirable than faradaic charge-injection materials because it creates or consumes no chemical species during stimulation. Moreover, the conductive polymer known as PEDOT, which delivers charges in a faradaic manner, has recently been studied as an alternative. Among the various materials, iridium oxide films have attracted significant attention from researchers due to their extraordinarily high charge-injection capacities. Iridium oxide has a high charge-injection capacity due to the reversible faradaic reactions it undergoes, involving reduction and oxidation processes between $\operatorname{Ir}^{3+}$ and $\mathrm{Ir}^{4+}$.

In order to form iridium oxide electrodes, various methods have been attempted, such as sputtering, activation, and electrodeposition. Sputtered iridium oxide film (SIROF) is formed by DC reactive sputtering using an iridium metal target under oxidizing plasma conditions. ${ }^{(11-15)}$ Activated iridium oxide film (AIROF) can be formed via an activation process in an aqueous electrolyte using iridium wires or sputtered iridium films. The activation is an oxidative process from iridium to iridium oxide by means of cycling between negative and positive potentials within the potential limits of water electrolysis. ${ }^{(6,16-18)}$ Electrodeposited iridium oxide film (EIROF) can be directly deposited from an iridium salt solution using pulsed voltage, DC voltage, or cyclic voltammetry.(19-23)

The different fabrication processes for iridium oxide film show different characteristics in terms of durability, fabrication complexity, or fabrication compatibility. For example, it was reported that AIROFs can be delaminated from an underlying iridium layer because uneven growth of the oxide layer introduces strain. ${ }^{(28,29)}$ In order to deposit SIROFs, in general, the substrate must be heated to a temperature above $400{ }^{\circ} \mathrm{C} \cdot{ }^{(30-32)}$ However, if the electrode substrate is made of polymers, 
the elevated temperature during sputtering is not suitable since the polymers are deformed near or above their melting temperature, which is typically around $300{ }^{\circ} \mathrm{C}$. In order to prevent the temperature increase of polymer substrates, intermediate cool-down steps were introduced during sputtering. ${ }^{(12)}$ However, this appeared to cause cracks in the SIROF layers during thermal lamination of LCP layers, which is an indispensable step in making monolithic and implantable neural electrodes from LCP. Unlike the SIROFs, the deposition of EIROFs is compatible with the thermal lamination process because the electrodeposition is generally performed after the fabrication processes are finished.

In this study, LCP-based EIROF electrodes were developed. These electrodes have the advantages of good flexibility and durability due to the use of a LCP substrate and a high charge storage capacity through their use of EIROF electrode sites. The use of EIROFs on LCP was previously mentioned very briefly. ${ }^{(20)}$ In this study, however, we established a fabrication process for LCP-based neural electrodes with optimized EIROF sites. The EIROFs were electrochemically formed with triangular waveforms and rectangular pulses to increase their adhesive stability and their charge storage capacity, respectively. ${ }^{(21)}$ We validated the characteristics of the fabricated LCP-based flexible neural electrodes with EIROF sites using electrochemical, mechanical, microscopic, and spectroscopic methods. Moreover, in vivo neural recording experiments were done to demonstrate the feasibility of the proposed EIROFs as electrode site materials.

\section{Materials and Methods}

\subsection{Electrode fabrication}

LCP-based neural electrodes were fabricated with multiple LCP films as shown in Fig. 1. The detailed fabrication procedures have been described previously. ${ }^{(2,9,43)}$ Briefly, as seed metal layers, $\mathrm{Ti}$ and $\mathrm{Au}$ were evaporated using an e-gun evaporator (Maestech Co., Ltd., ZZS550-2/ D, Pyungtaek, South Korea) on a LCP substrate layer (Kuraray Co., Ltd., Tokyo, Japan). In order to make the mask layer for gold electroplating, a photoresist (AZ 4620, Clariant, Muttenz, Switzerland) was patterned by photolithography using an aligner (SUSS MicroTec, MA6/BA6, Garching, Germany). After gold electroplating, the seed metal layers were wet-etched. As an insulating cover layer, another bare LCP film was laminated with the metal-patterned LCP layer via thermal pressing (Carver, Model 4122, Wabash, IN, USA). Laser ablation was then used to form a window to expose the electrode sites and the connecting pads using a laser micromachining process (DPSS Lasers Inc., Samurai UV Marking System, Santa Clara, CA, USA). After the final laser cutting process, the LCP-based neural electrode arrays with gold sites were fabricated. Lastly, iridium oxide was electrodeposited onto the gold sites. The detailed electrodeposition process is described in $\S 2.2$.

\subsection{Electrodeposition of iridium oxide}

Iridium oxide was electrodeposited from an aqueous solution of $4 \mathrm{mM} \mathrm{IrCl}_{4}$ in a supporting electrolyte of $40 \mathrm{mM}$ oxalic acid and $340 \mathrm{mM} \mathrm{K}_{2} \mathrm{CO}_{3}$, similar to the method described in previous studies. ${ }^{(21-23)}$ The first step in making the electrodeposition solution was to dissolve the $\mathrm{IrCl}_{4}$ in deionized water; this step took two hours. Into this solution, oxalic acid was dissolved. In another beaker, $\mathrm{K}_{2} \mathrm{CO}_{3}$ was dissolved in deionized water. The $\mathrm{K}_{2} \mathrm{CO}_{3}$ solution was then slowly 
(a) Fix LCP Film on Silicon Wafer

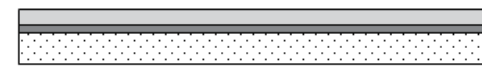

(b) Metal Seed Layers (Titanium and Gold) Deposition

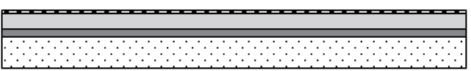

(c) Photolithography

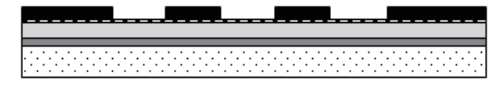

(d) Gold Electroplating

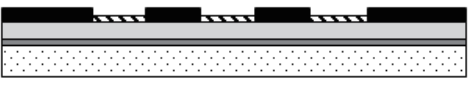

(e) Seed Layer Etching

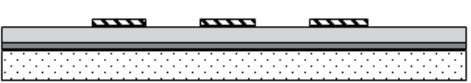

(f) Thermal Press Lamination

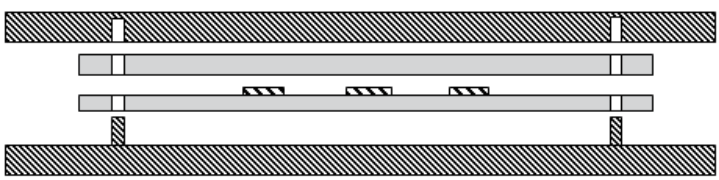

(g) Laser Ablation and Final Laser Cutting

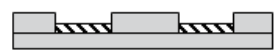

(h) Electrodeposition of Iridium Oxide
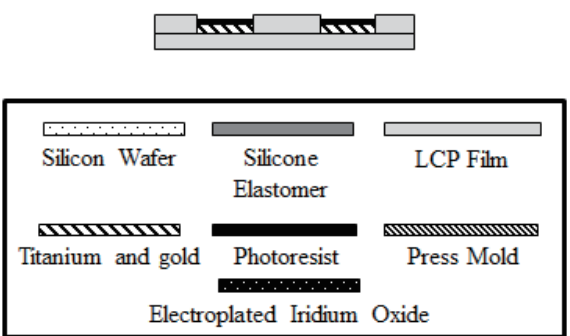

Fig. 1. Fabrication process of a LCP-based neural electrode: (a) LCP film was fixed onto a silicon wafer using a silicone elastomer, (b) metal seed layers of titanium and gold were evaporated on the LCP, (c) the photoresist was patterned for use as a mask during the gold electroplating process, (d) gold was electroplated, (e) the seed layers were wet-etched, (f) the metal-patterned LCP layer and the LCP cover layer were laminated together using a thermal press, and (g) with laser micromachining, the laser ablation and final laser cutting processes were done. Laser ablation was used to create a window to expose the electrode sites. (h) Lastly, iridium oxide was electrodeposited onto the gold sites.

added to the first solution. While mixing the two solutions, the $\mathrm{pH}$ was increasing to 10.3 . This electrodeposition solution was allowed to sit undisturbed for $48 \mathrm{~h}$ before the electrodeposition process. This step allows the establishment of an $\mathrm{Ir}^{3+} / \mathrm{Ir}^{4+}$ ratio at equilibrium in the solution, which is necessary to obtain stable EIROF films.

For the electrodeposition process, a three-electrode system was used consisting of the working electrode (gold electrode site on LCP substrate), a platinum counter electrode, and a silver/silver chloride $(3 \mathrm{M} \mathrm{KCl})$ reference electrode. The potential was controlled by a potentiostat (Solartron Analytical, 1287A, Farnborough, UK). The potential cycling was a combination of triangular waveforms and rectangular potential pulses. The basic electrodeposition protocol was 50 instances of the triangular waveform between the limits of $0.0 \mathrm{~V}$ and $0.55 \mathrm{~V}$ versus the $\mathrm{Ag} / \mathrm{AgCl}$ reference electrode at a $50 \mathrm{mV} / \mathrm{s}$ sweep rate followed by 1800 rectangular pulses at $0.55 \mathrm{~V}$. The rectangular pulses were applied at $1 \mathrm{~Hz}$ with a pulse duration of $0.5 \mathrm{~s}$. After the electrodeposition process, the electrode sites were rinsed with deionized water and dried by $\mathrm{N}_{2}$ gas. The triangular potential waveforms for the initial stages of the electrodeposition were utilized because they improve EIROF adhesion to substrates. The iridium oxide film was deposited onto the substrate during the potential cycling process, with triangular waveforms followed by rectangular pulses. To study the effects of the number of repetitions of the triangular waveforms and the rectangular potential pulses, we compared the electrochemical characteristics of iridium oxide films for different numbers of repetitions. 


\subsection{Electrochemical measurements}

The electrochemical impedance of the electrodes was characterized in a phosphate-buffered saline solution (Invitrogen Life Technologies, Gibco \#10010, Carlsbad, CA, USA) at pH 7.2 using an impedance analyzer (Solartron Analytical, 1287A, Farnborough, UK) in the three-electrode arrangement. The root-mean-square magnitude of the excitation voltage input was $10 \mathrm{mV}$ and the impedance was measured over a frequency range from $1 \mathrm{~Hz}$ to $100 \mathrm{kHz}$. Using the identical electrochemical cell, cyclic voltammetry was used to measure the cathodic charge storage capacity $\left(\mathrm{CSC}_{\mathrm{c}}\right)$. The electrochemical potential of the electrodes was swept from from -0.8 to $0.6 \mathrm{~V}$ vs an $\mathrm{Ag} / \mathrm{AgCl}$ reference electrode at a sweep rate of $100 \mathrm{mV} / \mathrm{s}$. The $\mathrm{CSC}_{\mathrm{c}}$ value was calculated using the time integral of the cathodic current during the cyclic voltammetry process. Electrochemical measurements were performed before and after the electrodeposition of iridium oxide to compare the electrochemical characteristics of the gold and the iridium oxide. The electrochemical measurements were done with electrodeposited iridium oxide films made with different numbers of repetitions of the triangular waveforms and the rectangular potential pulses.

The charge-injection limit of the fabricated EIROFs was also analyzed. The charge-injection limit is defined as the amount of charge that can be injected through the current stimulus pulse without water decomposition into hydrogen and oxygen. Cathodic current pulses with a pulse width of $1 \mathrm{~ms}$ and with varying amplitudes ranging from $0.1 \mathrm{~mA}$ to $1.3 \mathrm{~mA}$ were generated using a signal generator (World Precision Instruments, Sarasota, FL, USA). The cathodic current pulses were injected into the EIROF in a phosphate-buffered saline solution. Voltage data were acquired using a digital oscilloscope (Tektronix, DPO 4034, Beaverton, OR, USA) at a sampling rate of 2.5 GS/s.

\subsection{Surface morphology and mechanical stability}

The surface morphology of the EIROF was evaluated by atomic force microscopy (AFM) and scanning electron microscopy (SEM). AFM and SEM images of a gold electrode before the electrodeposition of iridium oxide were also acquired for comparison of the surface morphologies.

The mechanical stability of the EIROF was tested via a taping test and a sonication test. The taping test was done using a commercial adhesive tape (3M, Scotch Magic Tape 810) applied to a test area of the EIROF. Adhesion was considered adequate if the EIROF surface was not peeled off with the tape when the tape was detached. An ultrasonic wave of $40 \mathrm{kHz}$ was applied at $27^{\circ} \mathrm{C}$ for 10 min using an ultrasonic cleaner (Branson Ultrasonic Corporation, Bransonic 3510, Danbury, CT, USA). Impedance measurements and cyclic voltammetry measurements were performed before and after the stability tests to precisely examine the mechanical stability. SEM images and X-ray photoelectron spectroscopy (XPS) were also obtained before and after the stability tests to check the surface morphology and elementary composition.

\subsection{In vivo neural recording}

To verify the performance of the proposed EIROF as an electrode material, electrophysiological recordings were collected from animals with a neuropathic pain model. The electrical stimulation and monitoring were done simultaneously using a LCP-based neural electrode. The LCP-based neural electrode array has four channels; one of these was used for electrical stimulation and the others were used as recording electrodes. 
For the electrophysiological recording, Sprague-Dawley rats (male, 170-190 g, $n=15$ ) were used. In order to induce neuropathic pain in the rats, surgical procedures were used as described in our previous studies. ${ }^{(34,40,4445)}$ This study was conducted according to the guidelines of the ethics committee of the International Association for the Study of Pain ${ }^{(42)}$ and the Institution Animal Care and Use Committees of Yonsei University and Seoul National University.

After neuropathic pain was induced, electrical stimulation was performed while the local field potential (LFP) was monitored from the ventral posterolateral nucleus (VPL, AP $2.2 \mathrm{~mm}$ and ML $2.8 \mathrm{~mm}$ from bregma, and DV $6.0 \mathrm{~mm}$ from the skul1(33) ) to suppress neuropathic pain and monitor how the electrical stimulation alters the activity from the neighboring neurons. For the surgical procedures, the rats were anesthetized with pentobarbital sodium $(50 \mathrm{mg} / \mathrm{kg})$ and were fixed on a stereotaxic frame (Narishige, Tokyo, Japan). Their scalps were carefully opened and a small burr hole was made on the target area (VPL). A fabricated LCP-based EIROF microelectrode was inserted into the VPL. The electrode was firmly fixed on the skull, and the scalp was closed with sutures for permanent fixation of the electrode. One week later with animal care, the rats were anesthetized with urethane $(1.3 \mathrm{~g} / \mathrm{kg})$ for the LFP recordings. The electrodes were connected to an acquisition system consisting of a differential amplifier (Model 1700; A-M Systems, Sequim, WA, USA) and an AD converter (Micro 1401; Cambridge Electronic Design Limited, Cambridge, United Kingdom). Acquired signals were stored and displayed in a multi-channel continuous data acquisition and analysis package (Spike2, version 7, Cambridge Electronic Design Limited, Cambridge, UK).

To invoke pain by cold stimulus (cold allodynia), $0.1 \mathrm{~mL}$ of acetone was dropped onto the rat's contralateral hind paw. The detailed procedures for evoking cold allodynia and electrophysiological monitoring were presented in our previous report. ${ }^{(34)}$ Neuronal activity changes due to the electrical stimulation of the VPL were monitored simultaneously. Cathodic-first (biphasic) current pulses with an amplitude of $1.4 \mathrm{~mA}$, a pulse rate of $130 \mathrm{~Hz}$, and a pulse duration of $60 \mu$ s were delivered to the VPL.

\section{Results}

\subsection{Electrochemical measurements}

Figure 2 shows the LCP-based gold microelectrode before the electrodeposition of the iridium oxide and the electrodeposited iridium oxide film on the LCP-based microelectrode. The diameter of electrode sites was $300 \mu \mathrm{m}$ for all experiments. The EIROF was formed on the gold electrodes by potentiodynamic deposition using triangular potential waveforms followed by rectangular pulses. The $\mathrm{CSC}_{\mathrm{c}}$ and the electrochemical impedance at $1 \mathrm{kHz}$ were measured before and after the electroplating of the iridium oxide onto the gold layer. The cyclic voltammetry (CV) curves and the electrochemical impedance spectroscopy (EIS) results are shown in Fig. 3. The $\mathrm{CSC}_{\mathrm{c}}$ values were calculated from the $\mathrm{CV}$ curves, as explained in the methods section. Before the electrodeposition of the iridium oxide, the electrochemical impedance of the gold electrode sites at $1 \mathrm{kHz}$ and the cathodic charge storage capacity were measured and found to be $26.65 \pm 6.93 \mathrm{k} \Omega$ and $0.32 \pm 0.05$ $\mathrm{mC} / \mathrm{cm}^{2}$, respectively. Likewise, values of $1.41 \pm 1.1 \mathrm{k} \Omega$ and $24.19 \pm 6.21 \mathrm{mC} / \mathrm{cm}^{2}$ were measured after the electrodeposition of the iridium oxide (Fig. 3). The cathodic charge storage capacity was increased and the electrochemical impedance at $1 \mathrm{kHz}$ was decreased after the iridium oxide electroplating process, as expected. 

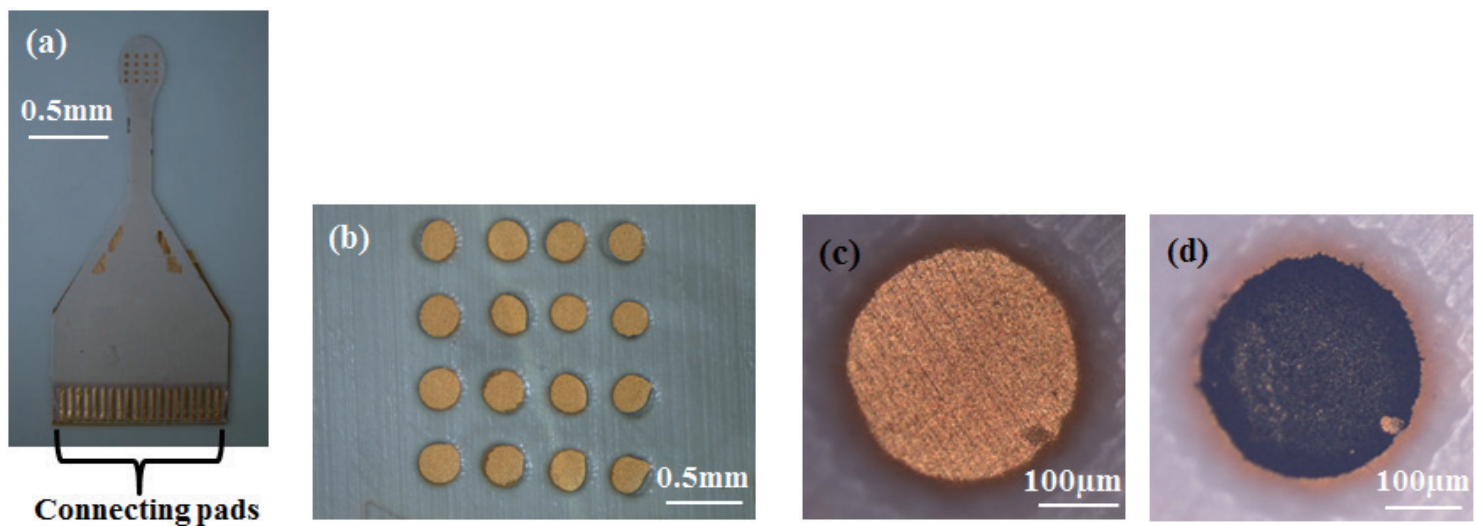

Fig. 2. (Color online) (a) LCP-based microelectrodes, (b) microelectrode sites formed with a radius of $150 \mu \mathrm{m}$, (c) gold electrode site, and (d) EIROF electrode site.

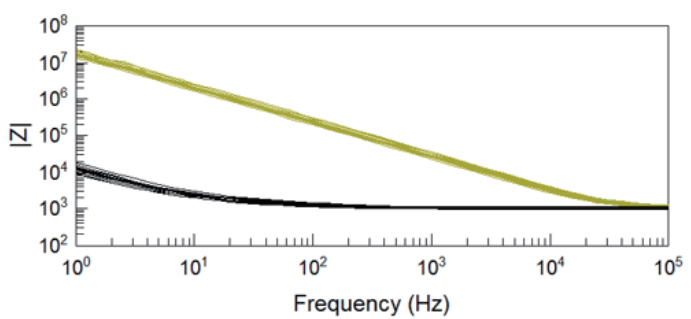

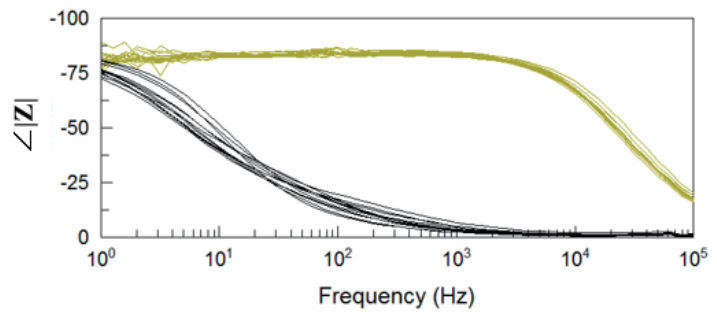

(a)

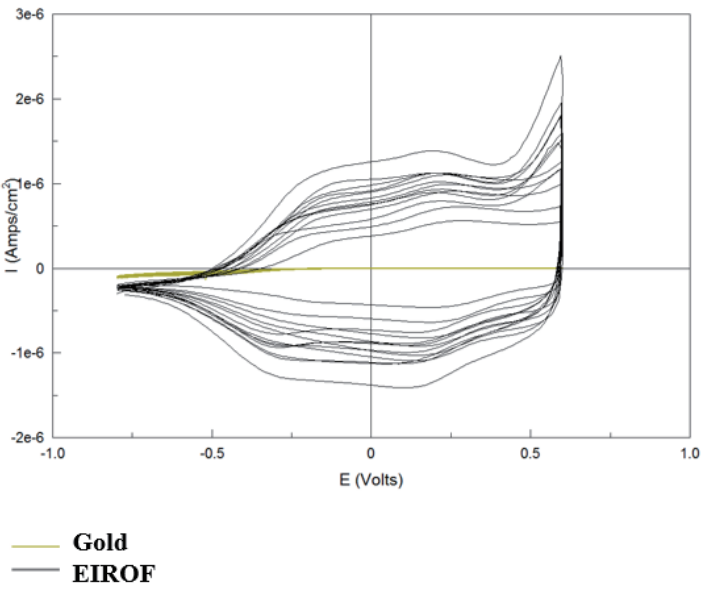

(b)

\begin{tabular}{lcc}
\hline & $\begin{array}{c}\text { Electrochemical impedence } \\
\text { at } 1 \mathrm{kHz}(\mathrm{k} \Omega)\end{array}$ & $\begin{array}{c}\text { Cathodic charge storage capacity } \\
\left(\mathrm{CSC}_{\mathrm{c}}\right)\left(\mathrm{mC}_{\mathrm{cm}}\right)^{2}\end{array}$ \\
\hline Gold & $26.65 \pm 6.93$ & $0.32 \pm 0.05$ \\
EIROF & $1.41 \pm 1.1$ & $24.19 \pm 6.21$ \\
\hline
\end{tabular}

Fig. 3. (Color online) Results showing the electrochemical characteristics of EIROFs with a radius of $150 \mu \mathrm{m}$; the EIROFs were created while varying the electrodeposition protocols, as noted in the methods section. (a) Electrochemical impedance results of gold and EIROFs and (b) CV curves of the gold and EIROFs.

To examine the effects of the number of repetitions of the rectangular potential pulse, the rectangular pulses were applied 1800,3600, and 5400 times. The cathodic charge storage capacity was increased as the number of rectangular pulses increased, as shown in Table 1 and Fig. 4. However, the electrochemical impedance was nearly identical, indicating that an increase in the number of rectangular potential pulses leads to a higher charge storage capacity in the EIROF. 
Table 1

Results showing the electrochemical characteristics with different numbers of repetitions of rectangular potential pulses (LCP-based microelectrodes with a radius of $150 \mu \mathrm{m}$ ) (number of triangular waveforms: 50).

\begin{tabular}{lcc}
\hline $\begin{array}{l}\text { Number of rectangular } \\
\text { pulses }\end{array}$ & $\begin{array}{c}\text { Electrochemical impedance } \\
\text { at } 1 \mathrm{kHz}(\mathrm{k} \Omega)\end{array}$ & $\begin{array}{c}\text { Cathodic charge storage } \\
\text { capacity }\left(\mathrm{CSC}_{\mathrm{c}}\right)\left(\mathrm{mC}_{\mathrm{cm}}\right)\end{array}$ \\
\hline 1800 & 1.44 & 11.13 \\
3600 & 1.13 & 20.24 \\
5400 & 1.10 & 25.58 \\
\hline
\end{tabular}

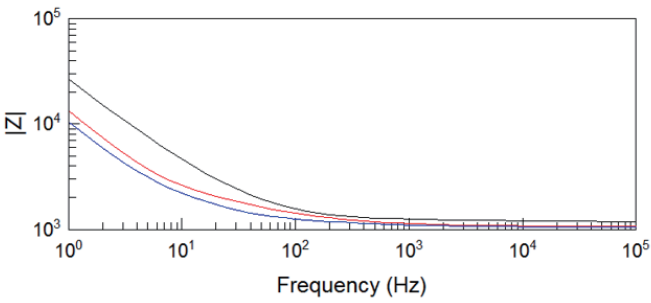

Number of Triangular waveforms :

50

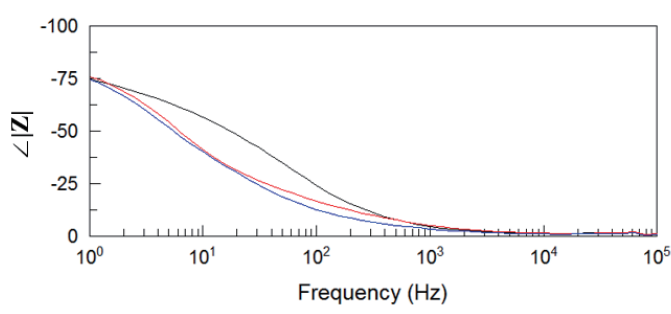

(a)
Number of Rectangular pulses : $\underline{1800} / \underline{3600} / \underline{5400}$

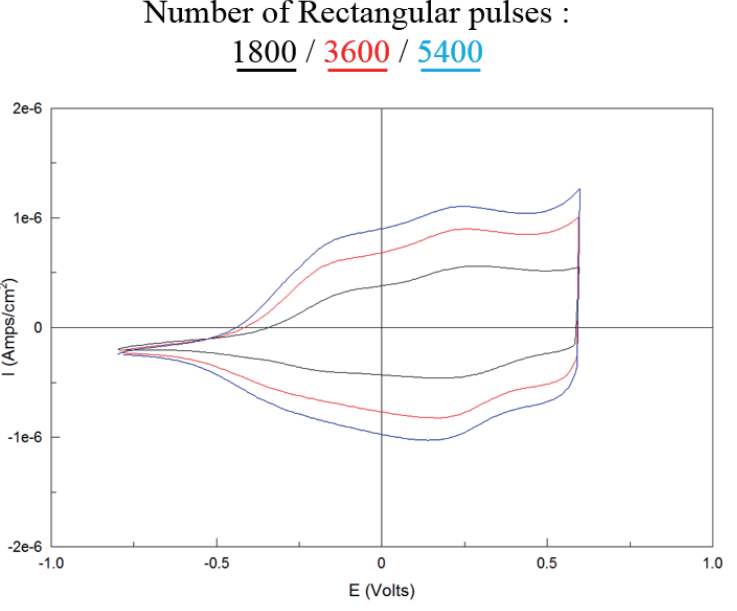

(b)

Fig. 4. (Color online) Results showing the electrochemical characteristics of EIROFs formed using 50 triangular waveforms and different numbers of rectangular potential pulses. The radius of the EIROFs was $150 \mu \mathrm{m}$. (a) Electrochemical impedance results of gold and EIROFs and (b) CV curves of gold and EIROFs [numbers of rectangular pulses: 1800 (black), 3600 (red), and 5400 (blue)].

However, the cathodic charge storage capacity and electrochemical impedance of EIROFs made with different numbers of repetitions of the triangular waveforms (50,100, 200, and 400 times) did not show a constant increasing or decreasing trend, as shown in Table 2 and Fig. 5. Because the triangular waveforms are known to affect the surface adhesion strength, ${ }^{(21)}$ the mechanical stability levels of EIROFs formed with different numbers of triangular waveforms were measured as described in the following section.

Figure 6(a) shows the $1 \mathrm{~ms}$ cathodal current pulses ranging from $0.1-1.3 \mathrm{~mA}$ used for chargeinjection limit analysis. During the current pulses flowing through the EIROF electrodes in PBS, the induced voltage transients were recorded and plotted in Fig. 6(b). Taking the lower voltage limit of the water window ( $-0.645 \mathrm{~V}$ vs the $\mathrm{Ag} / \mathrm{AgCl}$ reference electrode), the charge-injection limit of the fabricated EIROF was determined to be $1.27 \mathrm{mC} / \mathrm{cm}^{2}$. The charge storage capacity of the EIROF used for the charge-injection limit analysis was $23.54 \mathrm{mC} / \mathrm{cm}^{2}$. 
Table 2

Results showing the electrochemical characteristics for different numbers of repetitions of triangular waveforms (LCP-based microelectrodes with a radius of $150 \mu \mathrm{m}$ ) (number of rectangular pulses: 3600 ).

\begin{tabular}{lcc}
$\begin{array}{l}\text { Number of triangular } \\
\text { waveforms }\end{array}$ & $\begin{array}{c}\text { Electrochemical impedance } \\
\text { at } 1 \mathrm{kHz}(\mathrm{k} \Omega)\end{array}$ & $\begin{array}{c}\text { Cathodic charge storage } \\
\text { capacity }\left(\mathrm{CSC}_{\mathrm{c}}\right)\left(\mathrm{mC}_{\mathrm{cm}}\right)\end{array}$ \\
\hline 50 & 1.13 & 20.24 \\
100 & 1.09 & 22.89 \\
200 & 1.00 & 25.97 \\
400 & 1.15 & 24.26 \\
\hline
\end{tabular}
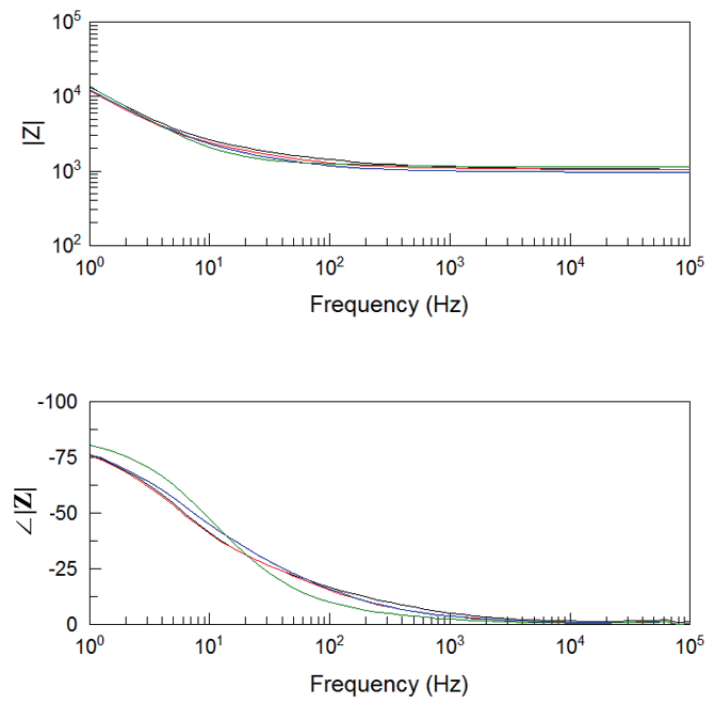

(a)
Number of Triangular waveforms :

$$
50 / 100 / 200 / 400
$$

Number of Rectangular pulses : 3600

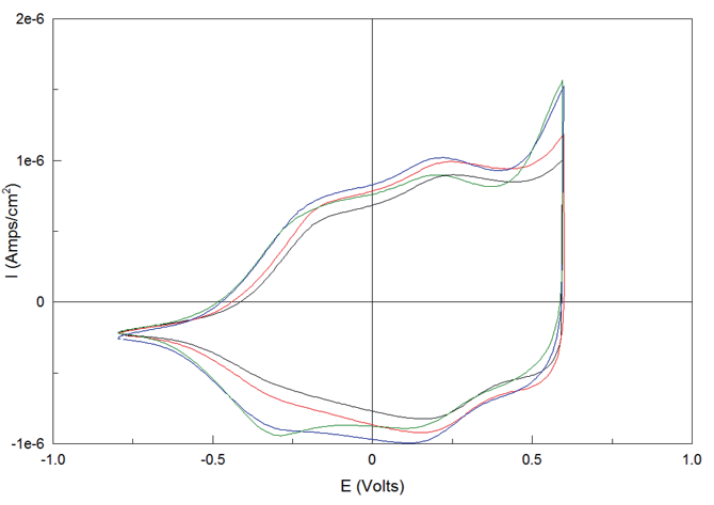

(b)

Fig. 5. (Color online) Results showing the electrochemical characteristics of EIROFs formed using different numbers of triangular waveforms and 1800 rectangular potential pulses. The radius of the EIROFs was $150 \mu \mathrm{m}$. (a) Electrochemical impedance results of gold and EIROFs and (b) cyclic voltammetry (CV) curves of gold and EIROFs [numbers of triangular waveforms: 50 (black), 100 (red), 200 (blue), and 400 (green)].

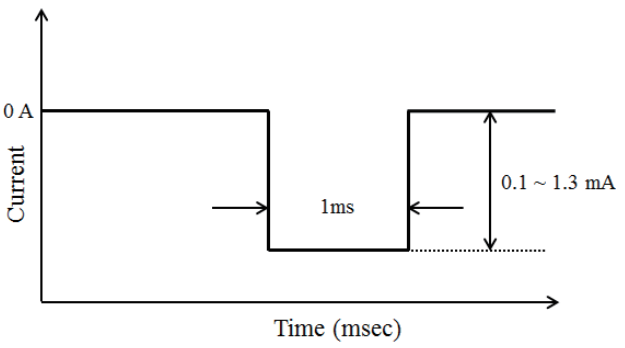

(a)

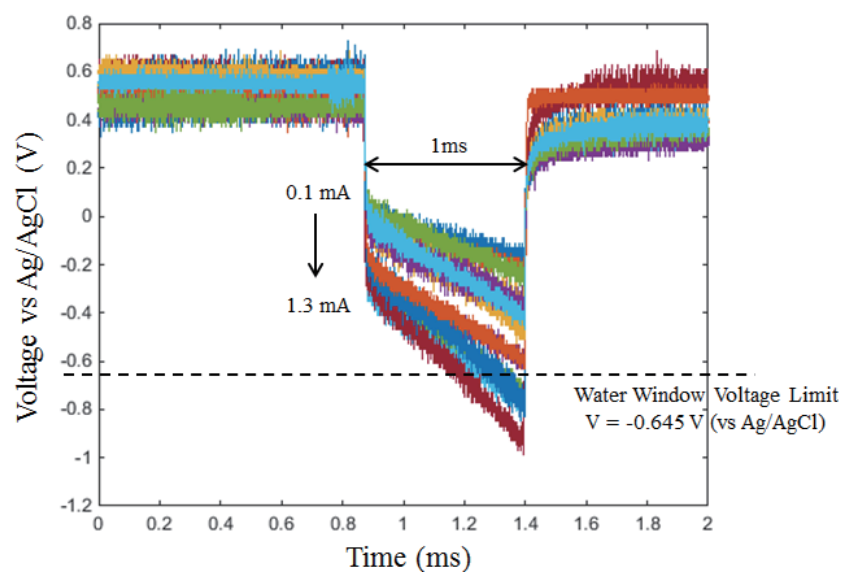

(b)

Fig. 6. (Color online) (a) Current pulse used for the charge-injection limit analysis and (b) voltage responses from the charge-injection limit analysis recorded from the EIROFs. 


\subsection{Surface morphology and mechanical stability}

In general, the effective surface of an electrode is proportional to the roughness of the electrode surface. When the roughness and porosity increase, the actual surface for an active chemical reaction between the electrode and the electrolyte also increases. In order to characterize the morphology of electrode surfaces, AFM and SEM images of the gold and EIROF were obtained and are depicted in Fig. 7 and Fig. 8, respectively. As shown in these images, the EIROF clearly has a rougher surface than gold, which facilitates effective neural stimulation with a large active surface area.

Mechanical stability of neural electrodes is required for implantable stimulating electrodes. To test the mechanical stability of the fabricated EIROF, a taping test and a sonication test were conducted. Taping tests were performed three times with tapes attached to the EIROF surfaces. The tape was placed on the surface of the substrate covering the electrode sites and then removed quickly by hand. Failures could occur at the weakest points of the electrode, such as the junction between the EIROF and LCP film. In the taping tests, however, no detachments between the EIROF and LCP interface were observed and the electrochemical characteristics were maintained (Fig. 9).

A triangular waveform in electrodeposition is known to improve EIROF adhesion to a substrate ${ }^{(21)}$. Accordingly, in order to investigate the effects of the triangular waveform, the EIROFs were deposited with different repetitions of the triangular waveform ranging from 50 to 400, and another stability test was performed, a sonication test, using an ultrasonic cleaner. Figure 10 and Table 3 show the electrochemical impedance at $1 \mathrm{kHz}$ and the $\mathrm{CSC}_{\mathrm{c}}$ before and after the sonication tests.

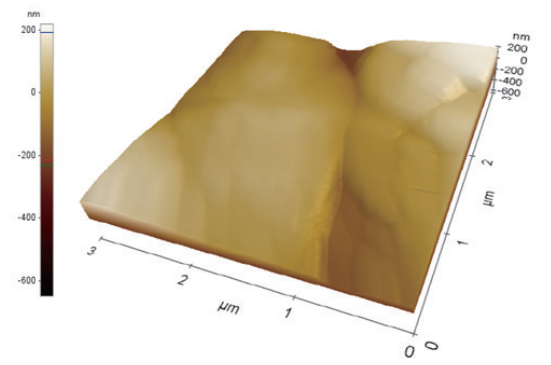

(a)

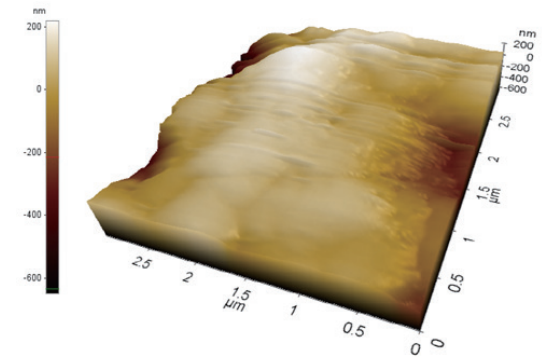

(b)

Fig. 7. (Color online) AFM images of (a) gold and (b) EIROF formed using 50 triangular waveforms and 5400 rectangular potential pulses.

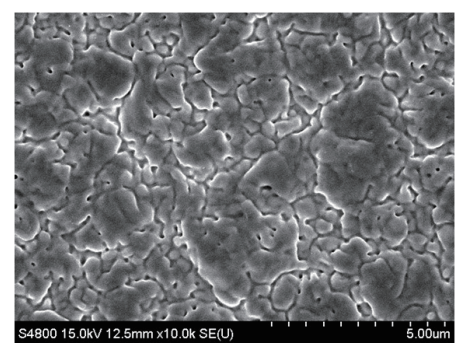

(a)

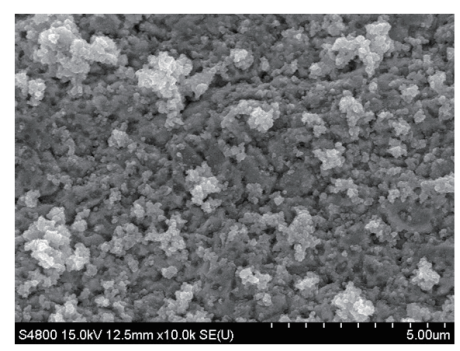

(b)

Fig. 8. SEM images of (a) gold and (b) EIROF formed using 50 triangular waveforms and 3600 rectangular potential pulses. 

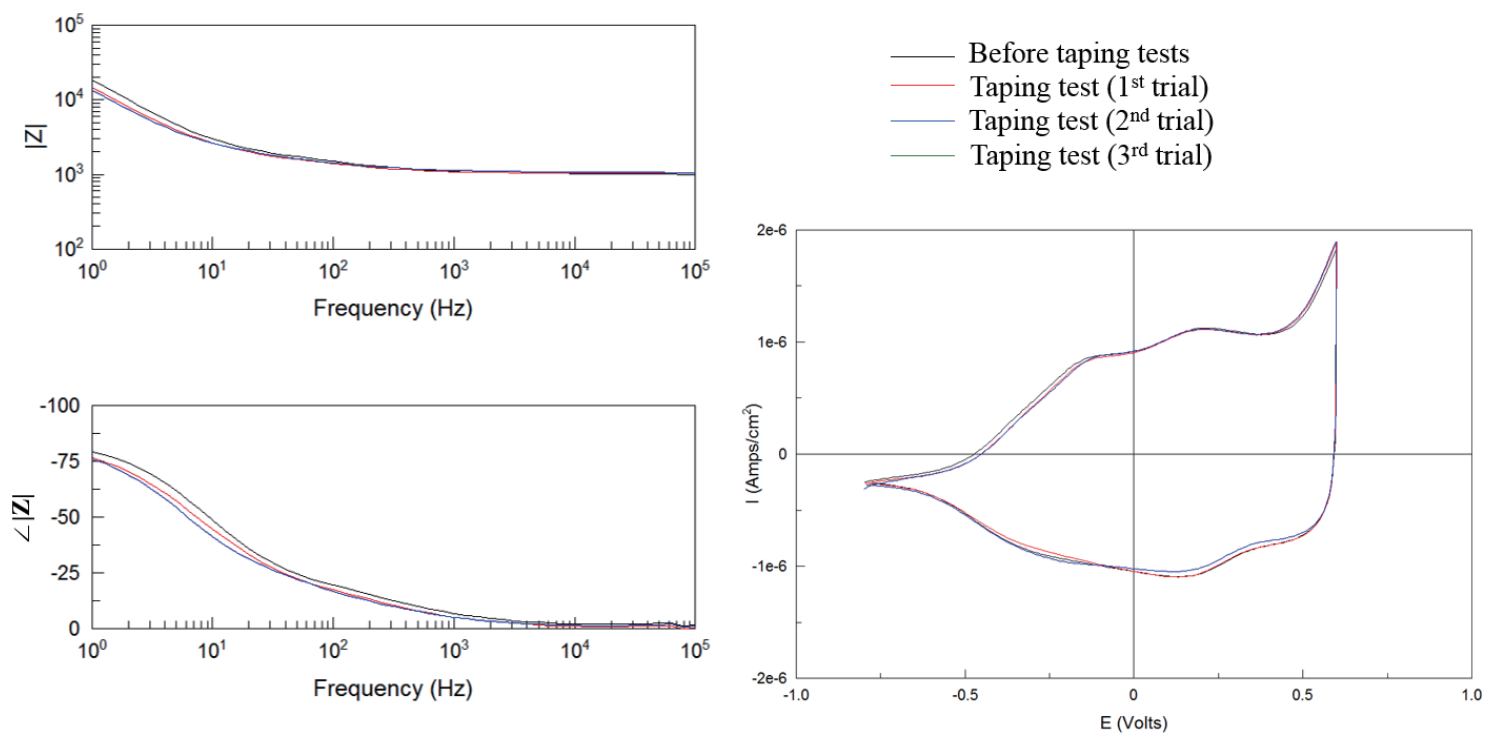

(a)

(b)

Fig. 9. (Color online) Results showing the electrochemical characteristics before and after the taping tests. The EIROF was made with 50 triangular waveforms and 5400 rectangular potential pulses. (a) Electrochemical impedance results and (b) cyclic voltammetry curves (radius of the EIROF, $150 \mu \mathrm{m}$ ) [black: before the taping tests, red: taping test (first trial), blue: taping test (second trial), green: taping test (third trial)].
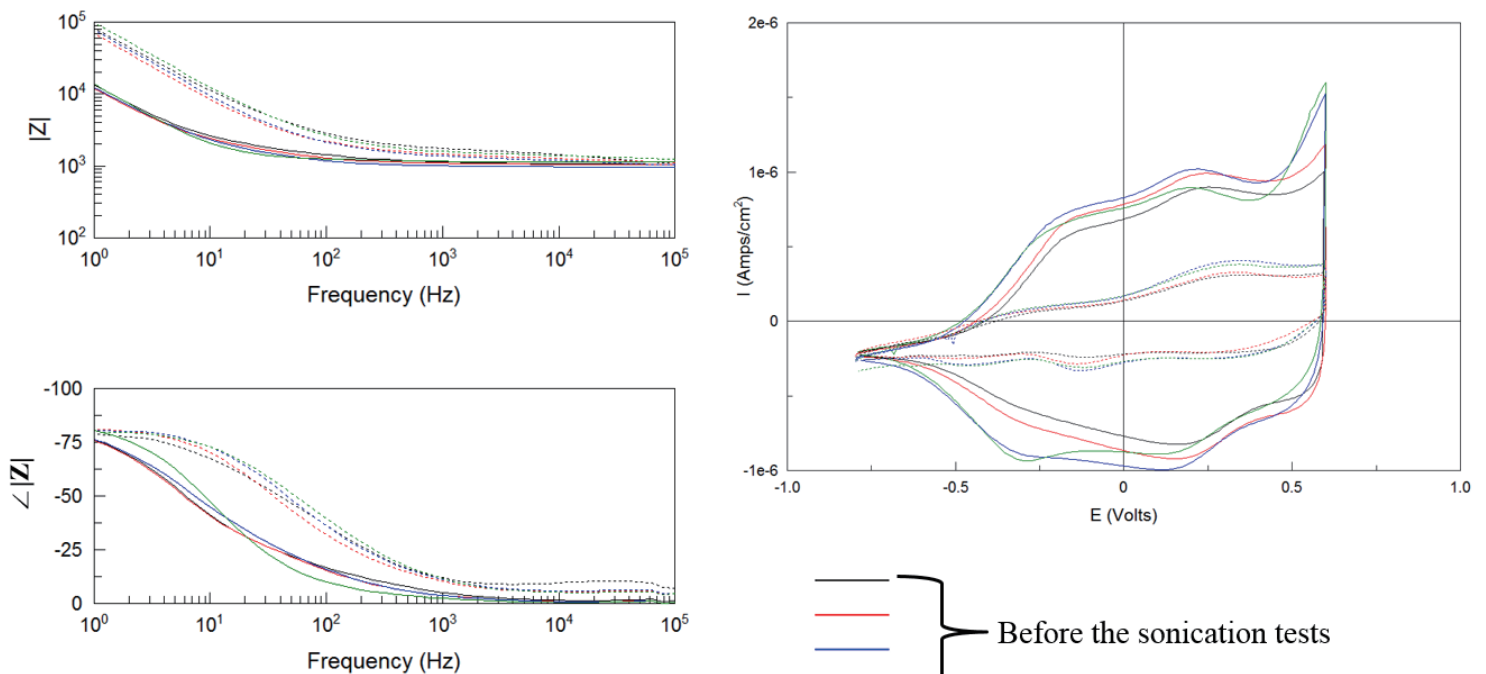

Number of Triangular waveforms :

$50 / 100 / 200 / 400$

Number of Rectangular pulses : 3600

(b)

Fig. 10. (Color online) Results showing the electrochemical characteristics before and after the sonication tests. The EIROFs were made with different numbers of triangular waveforms and with 3600 rectangular potential pulses. (a) Electrochemical impedance results and (b) cyclic voltammetry curves (radius of the EIROF, $150 \mu \mathrm{m}$ ) [numbers of triangular waveforms: 50 (black), 100 (red), 200 (blue), and 400 (green)] (straight lines: before the sonication tests, dotted lines: after the sonication tests). 
Table 3

Results showing the electrochemical characteristics for different numbers of repetitions of triangular waveforms before and after the sonication tests (LCP-based microelectrodes with a radius of $150 \mu \mathrm{m}$ ) (number of rectangular pulses: 3600$)$.

\begin{tabular}{lcccc}
\hline \multirow{2}{*}{$\begin{array}{l}\text { Number of triangular } \\
\text { waveforms }\end{array}$} & \multicolumn{2}{c}{$\begin{array}{c}\text { Electrochemical impedance } \\
\text { at } 1 \mathrm{kHz}(\mathrm{k} \Omega)\end{array}$} & \multicolumn{2}{c}{$\begin{array}{c}\text { Cathodic charge storage } \\
\text { capacity }\left(\mathrm{CSC}_{\mathrm{c}}\right)\left(\mathrm{mC} / \mathrm{cm}^{2}\right)\end{array}$} \\
\cline { 2 - 5 } & $\begin{array}{c}\text { Before the } \\
\text { sonication tests }\end{array}$ & $\begin{array}{c}\text { After the } \\
\text { sonication tests }\end{array}$ & $\begin{array}{c}\text { Before the } \\
\text { sonication tests }\end{array}$ & $\begin{array}{c}\text { After the } \\
\text { sonication tests }\end{array}$ \\
\hline 50 & 1.13 & 1.75 & 20.24 & 6.68 \\
100 & 1.09 & 1.45 & 22.89 & 6.88 \\
200 & 1.00 & 1.38 & 25.97 & 7.86 \\
400 & 1.15 & 1.59 & 24.26 & 8.36 \\
\hline
\end{tabular}

As shown in the results, the electrochemical impedance was increased and the $\mathrm{CSC}_{\mathrm{c}}$ value was decreased after the sonication tests. In Fig. 11, SEM images of EIROFs formed with different numbers of triangular waveforms before and after the sonication tests are shown. The white matter in this figure, presumably iridium particles, seemed to disappear significantly after the sonication tests regardless of the number of applied triangular waves. To study the effects of the applied triangular waveforms quantitatively, we analyzed the surfaces of EIROFs using XPS measurements (Fig. 12). According to the XPS results, the atomic percentages of the iridium in EIROFs made with 50 triangular waveforms decreased by $2.9 \%$, while the EIROFs created with 400 triangular waveforms showed a $1.6 \%$ decrease. Based on these results, the number of applied triangular waveforms affected the mechanical stability of the EIROFs.

\subsection{In vivo neural recording}

Using the developed EIROF as an electrode material, neuronal activities were recorded from the rat brain with neuropathic pain. The electrodes were positioned onto the VPL and the cold stimulusevoked neuronal responses were acquired. According to the previous study, pain can be evoked by a cold stimulus in the europathic pain (cold allodynia) model, generating ectopic discharges in the VPL. ${ }^{(34)}$ It was also reported that the cold pain can be suppressed by electrical stimulation on the VPL. ${ }^{(34)}$ In order to show the feasibility of the proposed electrode, electrical stimulation was delivered and the neural response was recorded via the electrodes in the neuropathic pain model while acetone evoked cold pain. ${ }^{(34,39)}$ The cold stimulus evoked a rapid change of the local field potential of the VPL [Fig. 13(a)]. However, the local field potential was scarcely changed by the electrical stimulation of the VPL [Fig. 13(b)]. Furthermore, the baseline amplitude of the field potential in the VPL was found to be higher after the electrical stimulation than in the resting state (without electrical stimulation). We assume that this higher background activity was generated by electrical stimulation. In some previous reports, it was found that pain suppression by electrical stimulation was maintained for at least $30 \mathrm{~min} .{ }^{(40,41)}$ In our recording results, the same tendency was demonstrated. The exaggerated baseline activity was reduced within minutes. 


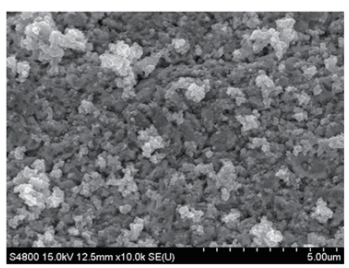

(a)

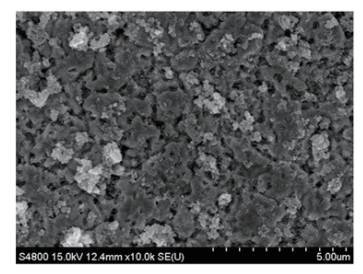

(b)

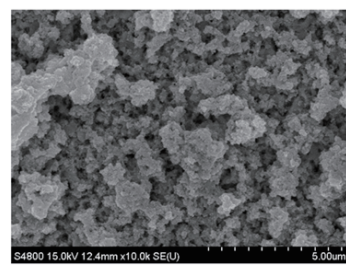

(c)

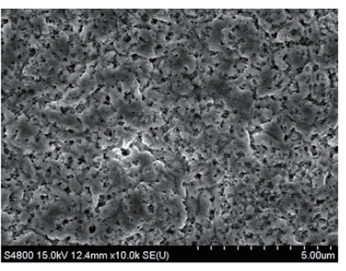

(d)

Fig. 11. SEM images of EIROFs: (a) and (b) are EIROFs made with 50 triangular waveforms and 3600 rectangular potential pulses, and (c) and (d) are EIROFs formed with 400 triangular waveforms and 3600 rectangular potential pulses. (a) and (c) are EIROFs before the sonication tests, and (b) and (d) are EIROFs after the sonication tests.

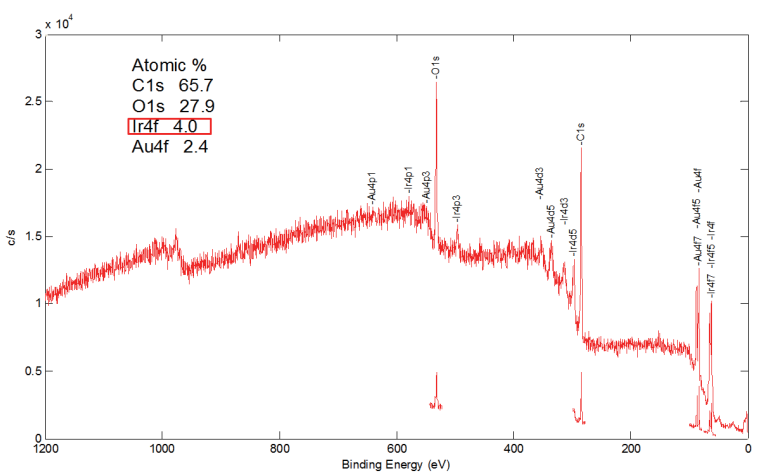

(a)

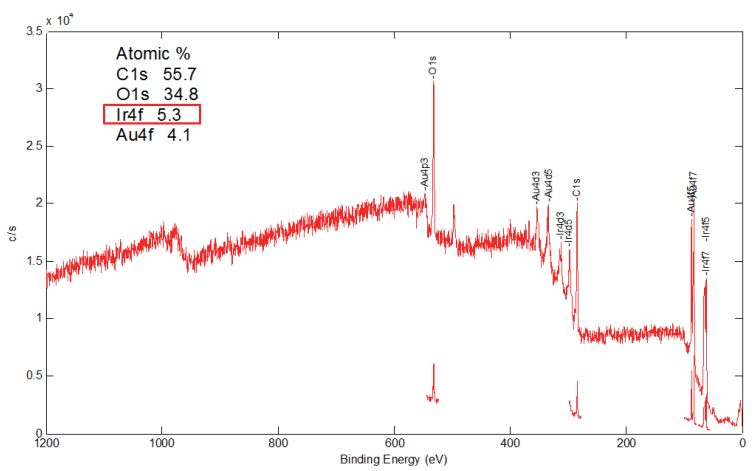

(c)

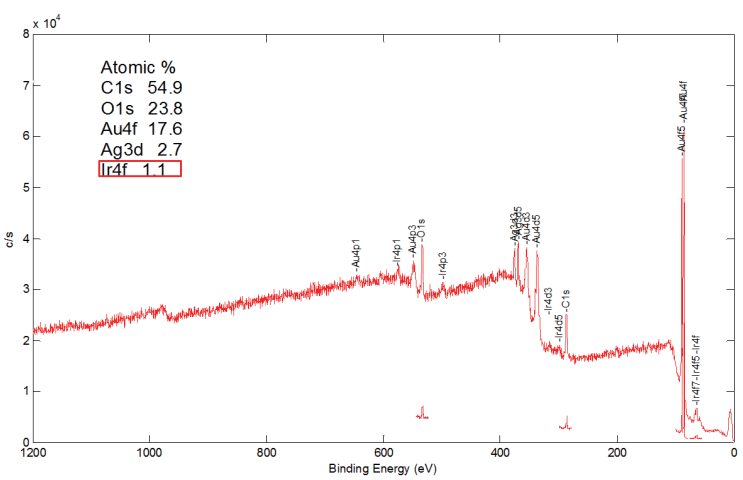

(b)

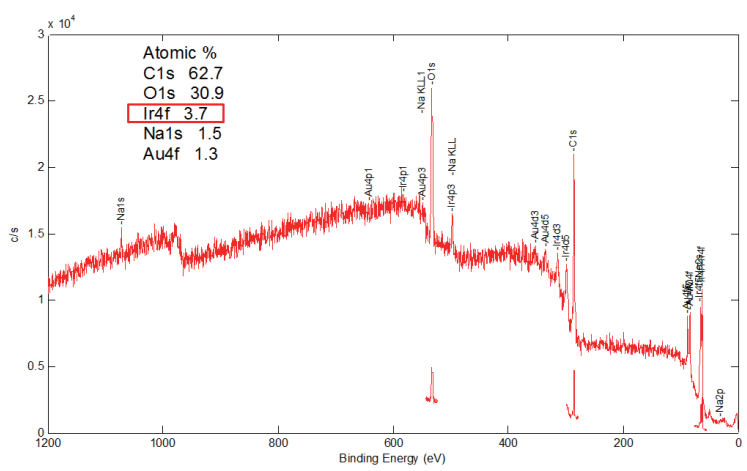

(d)

Fig. 12. (Color online) XPS measurement results: (a) and (b) are EIROFs made with 50 triangular waveforms and 3600 rectangular potential pulses, and (c) and (d) are EIROFs formed with 400 triangular waveforms and 3600 rectangular potential pulses. (a) and (c) are EIROFs before the sonication tests, and (b) and (d) are EIROFs after the sonication tests. 


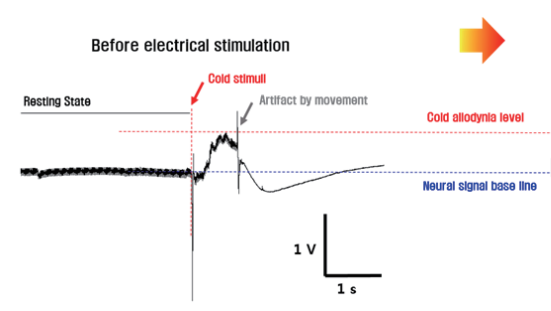

(a)

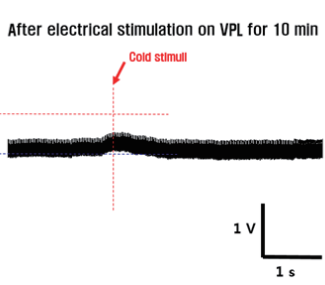

(b)

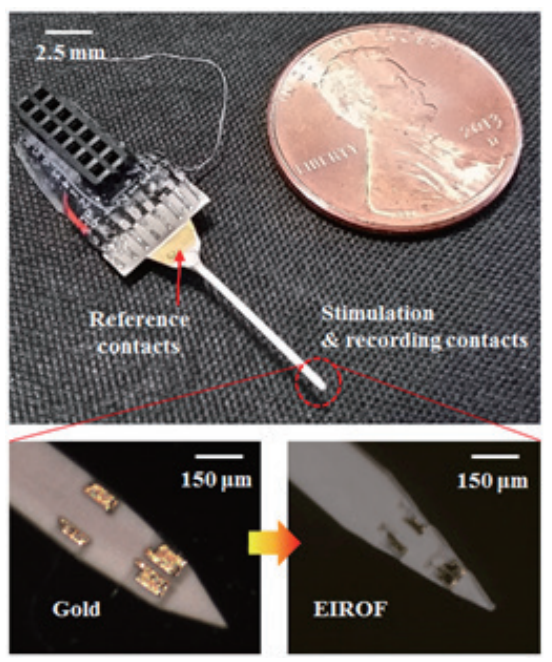

(c)

Fig. 13. (Color online) Results of in vivo neural recording experiments: (a) neural response before electrical stimulation and (b) changed neural response after electrical stimulation on VPL for 10 min. (c) A LCP-based deep brain stimulation electrode with EIROF electrode sites is shown.

\section{Discussion}

In this study, EIROF electrodes were formed on gold surfaces by application of triangular voltage waveforms followed by rectangular pulses in electrodeposition solution for iridium oxide. In order to investigate the effects of the triangular waveforms and the rectangular pulses on formation of EIROFs, different numbers of triangular waveforms and rectangular pulses were applied during the electrodeposition. Then the electrochemical and the mechanical characteristics of the formed EIROF electrodes were analyzed.

As the number of rectangular pulses increased, the charge storage capacity increased, whereas the electrochemical impedance was not affected. This was consistent with the results of SEM and XPS. SEM images confirmed that the surface morphologies of the EIROFs were similar even after different numbers of rectangular pulses as shown in Fig. 14. However, the XPS results indicated higher iridium content in EIROFs with the increased number of rectangular pulses (Fig. 15). As a result, the electrochemical impedance did not change because the porosity of the EIROF was not affected by the number of rectangular pulses, whereas the charge storage capacity increased due to the higher atomic content of iridium.

Unlike the rectangular pulses, the number of triangular waveforms did not affect either the electrochemical impedance or the charge storage capacity. However, it appears that the number of triangular waveforms is related to the mechanical stability of EIROFs. Even though the taping test did not show any meaningful change before and after the taping process, the sonication test resulted in different electrochemical characteristics and the surface morphologies of EIROFs after sonication as shown in Figs. 10 and 11. Regardless of the number of triangular waveforms, the 


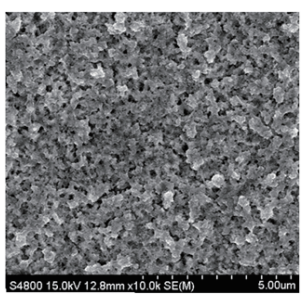

(a)

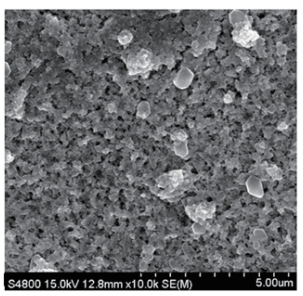

(b)

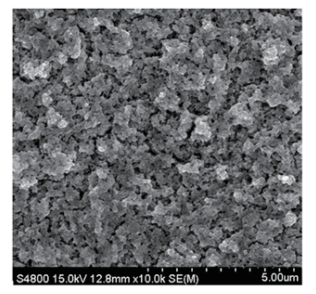

(c)

Fig. 14. SEM images of EIROFs made with different numbers of repetitions of rectangular pulses; the numbers of rectangular pulses were (a) 1800, (b) 3600, and (c) 5400 (number of triangular waveforms: 50).

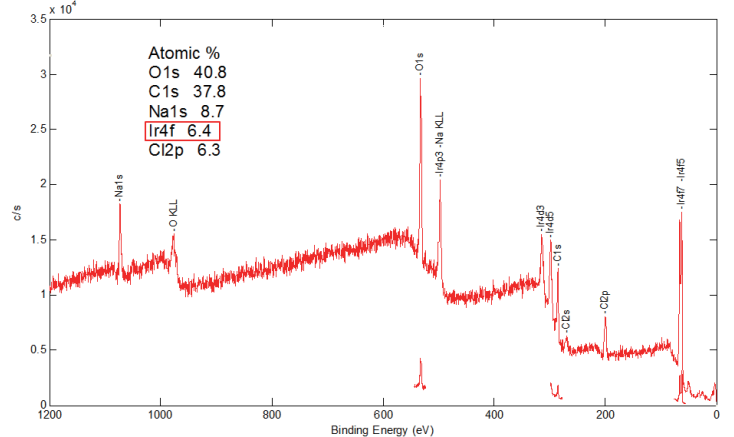

(a)

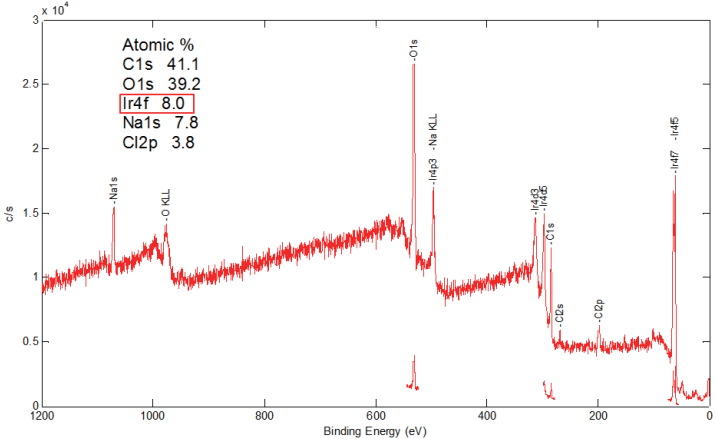

(b)

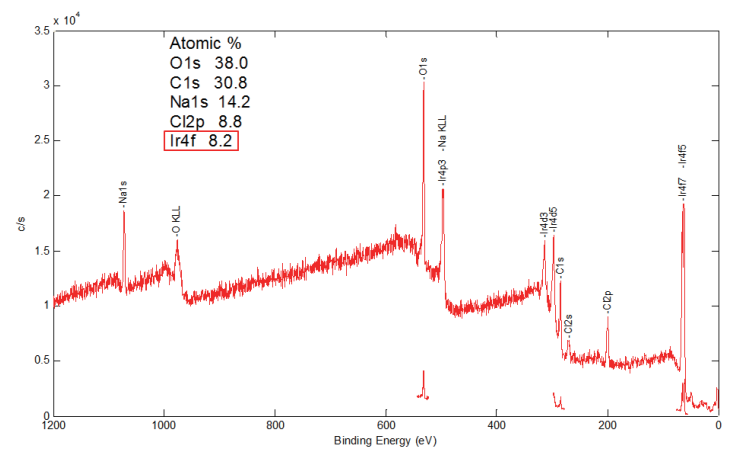

(c)

Fig. 15. (Color online) XPS measurements of EIROFs formed with different numbers of repetitions of the rectangular pulses; the numbers of rectangular pulses were (a) 1800, (b) 3600, and (c) 5400 (number of triangular waveforms: 50 ).

sonication obviously induced an increase in the electrochemical impedances and a decrease in the charge storage capacities as shown in Table 4. XPS analysis showed the results of different numbers of triangular waveforms in terms of the iridium compositions at the electrode surface. The composition of iridium decreased much more for the EIROFs formed using fewer triangular waveforms. It is likely that the triangular waveforms initiate the slower deposition of iridium oxide, which is advantageous for electroplating, especially on the different surface. The quantitative XPS 
Table 4

Averaged electrochemical characteristics of gold and EIROFs for various electrodeposition protocols, with the averaged electrochemical characteristics of EIROFs measured after the sonication tests (LCP-based microelectrodes with a radius of $150 \mu \mathrm{m}$ ) (The number of triangular waveforms was varied from 50, 100, 200, to 400 ; the number of rectangular pulses was varied from 1800,3600 , to 5400 ).

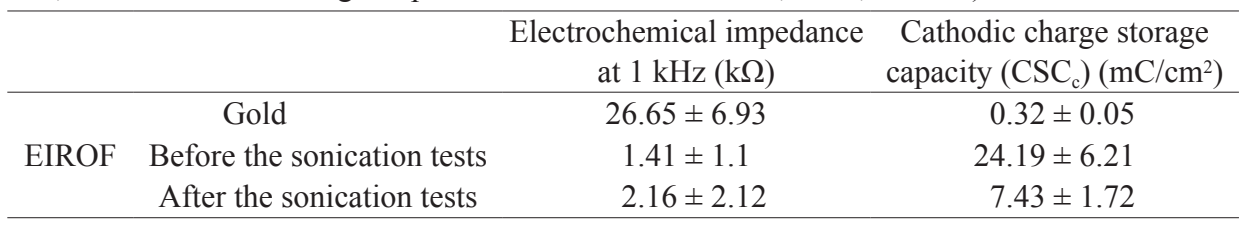

results support that an increase in the number of triangular waves can make the surfaces of EIROFs stronger and more stable.

The charge-injection limits for iridium oxide films (AIROF) on iridium wires are reportedly \pm 2 $\mathrm{mC} / \mathrm{cm}^{2}$ for the anodic current and $\pm 1 \mathrm{mC} / \mathrm{cm}^{2}$ for the cathodic, respectively. ${ }^{(46)}$ For iridium oxide films (SIROF) made from sputtered iridium metal, the charge-injection limit was reported to be 4-6 $\mathrm{mC} / \mathrm{cm}^{2}{ }^{(12)}$ The charge-injection limit of the electrodeposited iridium oxide film reported in this study was $1.27 \mathrm{mC} / \mathrm{cm}^{2}$. It may not be appropriate to directly compare the values from different research groups since the conditions for the measurements, such as electrolyte compositions, pulse width, reference electrode, electrode size, and to forth could be different. However, the proposed EIROF has a charge-injection limit comparable to the values in the literature. More importantly, the feasibility of the proposed EIROF electrode has been successfully shown using in vivo recording from rats with neuropathic pain. The neuronal activity of the VPL nucleus was found to increase rapidly when stimulated by cold, and it was suppressed by electrical stimulation of the VPL using the proposed microelectrodes with EIROFs. These findings reproduced those of previous studies of pain suppression by electrical stimulation, demonstrating the feasibility of the EIROF-fabricated microelectrodes. In fact, the proposed EIROF electrode has been recently adopted for LCP-based retinal implants. ${ }^{(43)}$

\section{Conclusion}

In general, effective electrical stimulation of the nerve system necessitates delivering a large amount of charge to the target neurons. To date, iridium oxide is one of the most effective materials due to its superior electrochemical characteristics for neural stimulation. In this study, we developed a neural electrode with EIROF using a flexible polymer substrate. The roles of triangular waveforms and rectangular pulses were confirmed in terms of the surface adhesion strength and charge-storage capacity. An increase in the number of triangular waves increased the mechanical stability of EIROF, while the increase in the number of rectangular pulses increased the charge storage capacity of EIROFs. Finally, the feasibility of a LCP-based microelectrode with the proposed EIROF electrode sites was verified in an in vivo neural recording experiment. The LCPbased neural electrodes with the proposed EIROF are expected to become a key technology for neural prostheses in the near future. 


\section{Acknowledgements}

This work was supported in part by the Pioneer Research Center Program through the National Research Foundation of Korea funded by the Ministry of Education, Science and Technology (NRF2009-0082961); in part by the Public Welfare \& Safety Research Program through the National Research Foundation of Korea funded by the Ministry of Education, Science and Technology (NRF-2010-0020851, NRF-2014R1A1A1A05003770); in part by the Center for Integrated Smart Sensors as GFP (CISS-2012M3A6A6054204); in part by an engineering-dentistry interdisciplinary research grant jointly funded by the College of Engineering and the College of Dentistry, Seoul National University; in part by the BK21 Plus Project, Department of Electrical and Computer Engineering, SNU, in 2015; in part by Business for Cooperative R\&D between Industry, Academy, and Research Institute funded Korea Small and Medium Business Administration in 2015 (C0275626); and in part by a grant to CABMC (Control of Animal Brain using MEMS Chip) funded by Defense Acquisition Program Administration (UD140069ID).

\section{References}

1 F. A. Spelman: Audiol. Neurotol. 11 (2006) 9.

2 K. S. Min, S. H. Oh, M. H. Park, J. Jeong, and S. J. Kim: Otol. Neurotol. 35 (2014) 8.

3 S. W. Lee, J.-M. Seo, S. Ha, E. T. Kim, H. Chung, and S. J. Kim: IOVS 50 (2009) 8.

4 J. D. Weiland and M. S. Humayun: Proceedings of the IEEE 96 (2008) 9.

5 J. A. Zhou, S. J. Woo, S. I. Park, E. T. Kim, J. M. Seo, H. Chung, and S. J. Kim: J. Biomed. Biotechnol. 2008 (2008) 10.

6 D. B. McCreery, T. G. H. Yuen, W. F. Agnew, and L. A. Bullara: Hear. Res. 62 (1992) 15.

7 A. L. Benabid: Curr. Opin. Neurobiol. 13 (2013) 11.

8 P. S. Motta and J. W. Judy: IEEE Trans. Biomed. Eng. 52 (2005) 11.

9 S. E. Lee, S. B. Jun, H. J. Lee, J. Kim, S. W. Lee, C. Im, H.-C. Shin, J. W. Chang, and S. J. Kim: IEEE Trans. Biomed. Eng. 59 (2012) 10.

10 S. F. Cogan: Annu. Rev. Biomed. Eng. 10 (2008) 35.

11 S. A. Kim, E. T. Kim, and S. J. Kim: J. Biomed. Eng. Res. 30 (2009) 6.

12 K. Wang, C.-C. Liu, and D. M. Durand: IEEE Trans. Biomed. Eng. 56 (2009) 9.

13 T. Stieglitz, H. Beutel, M. Schuettler, and J.-U. Meyer: Biomedical Microdevices 2 (2000) 12.

14 S. F. Cogan, J. Ehrlich, T. D. Plante, A. Smirnov, D. B. Shire, M. Gingerich, and J. F. Rizzo: J. Biomed. Mater. Res. B. Appl. Biomater. 89 (2009) 9.

15 K. S. Kang and J. L. Shay: J. Electrochem. Soc. 130 (1983) 4.

16 S. F. Cogan: Conf. Proc. IEEE Eng. Med. Biol. Soc. (2006) 4.

17 X. Beebe and T. L. Rose: IEEE Trans. Biomed. Eng. 35 (1988) 5.

18 D. C. Rodger, A. J. Fong, W. Li, H. Ameri, A. K. Ahuja, C. Gutierrez, I. Larvov, H. Zhong, P. R. Menon, E. Meng, J. W. Burdick, R. R. Roy, V. R. Edgerton, J. D. Weiland, M. S. Humayun, and Y.-C. Tai: Sens. Actuators, B: Chem. 132 (2008) 12.

19 J. D. Weiland, S. Cogan, and M. S. Humayun: Conf. Proc. IEEE Eng. Med. Biol. Soc. (2004) 7.

20 S. Corbett, J. Jetterl, and T. Johnson: Mater. Res. Soc. Symp. Proc. 926 (2006) 6.

21 R. D. Meyer, S. F. Cogan, T. H. Nguyen, and R. D. Rauh: IEEE Trans. Neural. Syst. Rehabil. Eng. 1 (2001) 10.

22 K. Yamanaka: Jpn. J. Appl. Phys. 28 (1989) 6.

23 S. A. M. Marzouk, S. Ufer, and R. P. Buck: Anal. Chem. 70 (1998) 8.

24 M. H. Hassan, V. Chodavarapu, and S. Musallam: Sensors 8 (2008) 13.

25 C. Hassler, T. Boretius, and T. Stieglitz: J. Polymer Sci.: Part B: Polymer Phys. 46 (2011) 16.

26 X. Wang, J. Engel, and C. Liu: J. Micromech. Microeng. 13 (2003) 6.

27 S. W. Lee, K. S. Min, J. Jeong, J. Kim, and S. J. Kim: IEEE Trans. Neural. Syst. Rehabil. Eng. 58 (2011) 9.

28 E. Slavcheva, R. Vitushinsky, W. Mokwa, and U. Schnakenberg: J. Electrochem. Soc. 151 (2004) 12. 
29 S. F. Cogan, A. A. Guzelian, W. F. Agnew, T. G. H. Yuen, and D. B. McCreery: J. Neurosci. Methods 137 (2004) 10.

30 R. H. Horng, D. S. Wuu, L. H. Wu, and M. K. Lee: Thin Solid Films 373 (2000) 4.

31 T. J. Park, D. S. Jeong, C. S. Hwang, M. S. Park, and N. S. Kang: Thin Solid Films 471 (2005) 7.

32 A. Oliveira-Sousa, M. A. S. Silva, S. A. S. Machado, L. A. Avaca, and P. Lima-Neto: Electrochim. Acta. 45 (2000) 7.

33 G. Paxinos and C. Watson: The rat brain in stereotaxic coordinates (Academic Press, San Diego, 1998).

34 J. Kim, S. E. Lee, K. S. Min, H. H. Jung, J. E. Lee, S. J. Kim, and J. W. Chang: J. Neurosci. Res. 91 (2013) 8.

35 D. N. Rushton: Disability Rehabil. 24 (2002) 9.

36 P. Shirvalkar, M. Seth, N. D. Schiff, and D. G. Herrera: Proc. Natl. Acad. Sci. 103 (2006) 6.

37 A. F. Da Silva, C. Granziera, J. Snyder, and N. Hadjikhani: Neurology 69 (2007) 6.

38 P. Blomstedt, U. Sandvik, A. Fytagoridis, and S. Tisch: Neurosurgery 64 (2009) 10.

39 B. H. Lee, R. Won, E. J. Baik, S. H. Lee, and C. H. Moon: Neuroreport 11 (2000) 5.

40 J. Kim, K. S. Min, S. E. Lee, S. J. Kim, and J. W. Chang: Neurol. Sci. 33 (2012) 6.

41 J. M. Lucas, Y. Ji, and R. Masri: Pain 152 (2011) 10.

42 M. H. Zimmermann: Xylem Structure and the Ascent of Sap. (Springer-Verlag, Berlin-Heidelberg-New YorkTokyo 1983) p. 143.

43 J. Jeong, S. H. Bae, K. S. Min, J-M. Seo, H. Chung, and S. J. Kim: IEEE Trans. Biomed. Eng. 62 (2015) 8.

44 J. Kim, S. E. Lee, J. Shin, H. H. Jung, S. J. Kim, and J. W. Chang: J. Korean Neurosurg. Soc. 57 (2015) 6.

45 K. S. Min, C. J. Lee, S. B. Jun, S. E. Lee, J. Shin, J. W. Chang, and S. J. Kim: Neuromodulation 17 (2014) 10.

46 W. F. Agnew and D. B. McCreery: Neural Prostheses: Fundamental Studies. Englewood Cliffs, NJ: PrenticeHall, 1990. 